\title{
Convex Saturated Particle Filter
}

\author{
Paweł Mirosław Stano*, Arnold J. den Dekker, Zsófia Lendek, Robert Babuška
}

\begin{abstract}
In many systems the state variables are defined on a compact set of the state space. To estimate the states of such systems, the constrained particle filters have been used with some success. The performance of the standard particle filters can be improved if the measurement information is used during the importance sampling of the filtering phase. It has been shown that the particles obtained in such a way approximate the true state of the system more accurately. The measurement is incorporated into the filtering algorithm through a userspecified detection function, which aims to detect the saturation as it occurs. The algorithm derived from the aforementioned principle is called the Saturated Particle Filter (SPF). In our previous work we have derived a complete SPF framework for the class of systems with one-dimensional constraints. In this paper we derive a novel Convex SPF that extends our method to multidimensional systems with convex constraints. The effectiveness of the new method is demonstrated using an illustrative example.
\end{abstract}

\section{INTRODUCTION}

Dynamic filters are commonly used in various engineering problems that are modeled by a Stochastic Dynamical System (SDS). When a SDS has linear dynamics and additive Gaussian noises it is well known that the optimal estimator, in the mean square error (MSE) sense, is the Kalman Filter (KF) [1], [2]. In case of nonlinear and/or non-Gaussian noises, in general, the optimal solution is computationally intractable. Thus, one needs to rely on suboptimal solutions. Several versions of the KF that give a suboptimal solution have been developed to address the nonlinear filtering problem [3]. These include, among others, the Extended Kalman Filter [2], the Unscented Kalman Filter [2], [4], [5], and the Gauss-Hermite Filter [6], [7]. All of these methods are classified as parametric, i.e., they solve a finite dimensional estimation problem. Parametric filters are simple to implement and very effective when applied to stochastic processes that can be accurately approximated by Gaussian processes. However, in case of highly nonlinear and nonGaussian dynamical systems, their performance deteriorates.

As an alternative to parametric methods, the nonparametric Particle Filter (PF) has been proposed [2], [8] as a tool to solve a general filtering problem. This algorithm aims to estimate a Probability Density Function (PDF) of the state rather than a point statistic of the state. Thus, the estimation problem becomes infinite dimensional.

The PF approximates a PDF of the state of the system by a set of points which are obtained by utilizing

P. M. Stano, A. J. den Dekker, R. Babuška are with the Delft Center for Systems and Control Delft University of Technology, Mekelweg 2, 2628 CD Delft, The Netherlands, P.M.Stano@tudelft.nI

Zsófia Lendek is with the Department of Automation, Technical University of Cluj-Napoca, Memorandumului 28, 400114 Cluj-Napoca, Romania. the Importance Sampling method [8], and then weighted according to the Bayes rule. The PF is based on a Monte Carlo approximation and it has been proven [9] that under mild technical assumptions the PF-based PDF converges to the true posterior PDF as the number of samples grows. However, for highly nonlinear and non-Gaussian systems the $\mathrm{PF}$ might require a large number of samples to achieve an accurate estimate. This makes the algorithm computationally expensive, especially in high-dimensional systems, and as a consequence, it limits its on-line applicability. It has been noted [10], [11], [8], [2] that the choice of the importance sampling density is a crucial step towards reducing the computational costs of PFs, and therefore making the method more feasible for on-line applications.

The properties of the PF have been extensively studied in recent years [2], [8], [9], and many versions of the PF have been developed for specific types of problems [8], [12], [13], [14]. In particular, state estimation of a Constrained Stochastic Dynamical System (CSDS) attracted much attention [15], [16], [12], [13], [14], [17]. The CSDS is a system for which, at each time step $k$, at least one of the state variables is restricted to a compact set. These systems are frequently met both in industrial applications [18], [19], and in theoretical research [12], [20], [15]. To estimate the states of the CSDS one can use the constrained PF [16], [12], [13], [14]. This method produces a state estimate that does not violate the physical constraints of the system that is achieved either by discarding unsuitable particles [16], [13], or by projecting them on a boundary of the constraint region [12], [14]. The latter approach is specially suitable for systems characterized by PDFs that are singular (discontinuous) at the boundary of the constraint region. The boundary of such a set is denoted by the saturation region and the particles located at this boundary are called the saturated particles. The system defined by this type of PDF is called [17] the Saturated Stochastic Dynamical System (SSDS), which is a special class of the CSDS.

An efficient method for estimating the states of SSDSs, the Saturated Particle Filter (SPF), has been proposed in [17]. The SPF combines the projection approach of [12] with a novel sampling method that effectively detects the saturation moment, and forces the particles to rapidly jump to that part of the state space which is close to the saturation region. Such sampling is obtained by designing an importance density function that makes use of both the measurement and the knowledge of the system constraints. Several resampling methods suitable for SPF have been derived in [21]. Furthermore, also in [21], it has been shown that the SPF asymptotically converges to the true posterior PDF. 
The SPF proposed in [17] has been derived for a special class of SSDSs, namely for systems that allow only one-dimensional saturation, i.e., if $x_{k}=$ $\left[\begin{array}{lll}x_{k}(1) & \cdots & x_{k}(n)\end{array}\right]^{T}$ is an $n$-dimensional state variable then only one of the variables $x_{k}(1), \cdots, x_{k}(n)$ can be saturated. The framework of [17] can be easily extended to systems with multidimensional saturations provided that the saturated variables are independent. However, the extension for general multidimensional SSDSs is not straightforward. In this paper we aim to fill this gap in the SPF framework by deriving the Convex Saturated Particle Filter (CSPF), which is applicable to multidimensional systems with convex constraints imposed on the states. The assumption of the convexity of the constraints, from the practical perspective, is not very restrictive. In fact a stronger condition of linear constraints is commonly met in the literature [15], [19], [14], [22].

The paper is organized as follows: in Section II-A the mathematical framework of the Convex Saturated Stochastic Dynamical System is defined and the estimation problem is stated. In Section II-B the standard solution to the previously formulated problem is given. The novel Convex Saturated Particle Filter is derived in Section III. In Section IV the new filter is compared with the benchmark method. Section V concludes the paper.

\section{PRELIMINARIES}

This section contains preliminaries and basic motivations for the development of the Saturated Particle Filter. First, the mathematical framework that we use to model saturated processes is defined. Next, the basic facts about Particle Filtering are recalled.

\section{A. System Definition}

For the $n_{x}$-dimensional state space $\mathcal{X}$, the $n_{u}$-dimensional input space $\mathcal{U}$, and the $n_{y}$-dimensional observation space $\mathcal{Y}$, the generic stochastic dynamical system is defined by:

Definition 1 (Stochastic Dynamical System): The Stochastic Dynamical System (SDS) is defined as a pair $\left\{\left(x_{k}, y_{k}\right)\right\}_{k=0}^{+\infty}$ composed of dependent discrete-time stochastic processes $\left\{x_{k}\right\}_{k=0}^{+\infty}$, and $\left\{y_{k}\right\}_{k=0}^{+\infty}$ that evolve according to:

$$
\begin{aligned}
x_{k+1} & =f_{k}\left(x_{k}, u_{k}, w_{k}\right), \\
y_{k} & =h_{k}\left(x_{k}, u_{k}\right)+v_{k}, \\
x_{0} & \sim p_{0}(\cdot),
\end{aligned}
$$

where $w_{k}$ and $v_{k}$ are mutually independent Gaussian variables that take values in the state space $\mathcal{X}$ and the observation space $\mathcal{Y}$ respectively, $f_{k}: \mathcal{X} \times \mathcal{U} \times \mathcal{X} \rightarrow \mathcal{X}$ is a (possibly nonlinear) function that describes the state evolution, $h_{k}$ : $\mathcal{X} \times \mathcal{Y} \rightarrow \mathcal{Y}$ is a (possibly nonlinear) function that establishes the observation model, $u_{k}$ denotes the deterministic input at time step $k$, and $p_{0}$ is a PDF of the initial state $x_{0}$, which is independent of the noises $w_{k}$ and $v_{k}$.

The stochastic process defined by (1) is Markovian, which allows for the recursive estimation of the state of the system.
A saturated system is obtained by imposing extra conditions on the system defined by (1). Namely, we consider systems that satisfy the following definition:

Definition 2 (Saturated SDS): Let $\left\{\left(x_{k}, y_{k}\right)\right\}_{k=0}^{+\infty}$ be a SDS defined by (1). The sequence of tuples $\left\{\left(x_{k}, y_{k}\right)\right\}_{k=0}^{+\infty}$ is called a Saturated Stochastic Dynamical System (SSDS) if for each $k \geq 1$ there exists a precompact [23] open set $C_{k} \subset \mathcal{X}$ such that:

1) the support of the conditional distribution of the state $x_{k+1}$ given $x_{k}$ and $u_{k}$ is contained within the closure of the $C_{k}$ (denoted by $\left.\overline{C_{k}}\right)$, i.e.,

$$
\mathbb{P}\left(x_{k+1} \in \overline{C_{k}} \mid x_{k}, u_{k}\right)=1,
$$

2) the transition probability of the state $x_{k+1}$ belonging to $\partial C_{k}$, given $x_{k}$ and $u_{k}$, is positive, i.e.,

$$
\mathbb{P}\left(x_{k+1} \in \partial C_{k} \mid x_{k}, u_{k}\right)>0,
$$

where $\partial C_{k}$ denotes the topological boundary of the set $C_{k}$.

In what follows we consider the SSDSs such that for each $k \geq 1$ the constraint region of the variable $x_{k+1}$, which will be dependent on the previous state $x_{k}$ and the previous input $u_{k}$, is a convex set. The precise conditions that such systems need to satisfy are listed in Definition 3.

Definition 3 (Convex SSDS): Let $\Sigma_{\mathcal{X}}$ be the collection of measurable subsets of $\mathcal{X}$. The $\operatorname{SSDS}\left\{\left(x_{k}, y_{k}\right)\right\}_{k=0}^{+\infty}$ is called the Convex Saturated Stochastic Dynamical System (CSSDS) if there exist a function $C: \mathcal{X} \times \mathcal{U} \rightarrow \Sigma_{\mathcal{X}}$ and measurable functions $\tilde{f}_{k}: \mathcal{X} \times \mathcal{U} \times \mathcal{X} \rightarrow \mathcal{X}$ such that for each $k \geq 1$ the following hold:

1) for every convex $A \in \Sigma_{\mathcal{X}}$ the set $h_{k}(A)$ is convex,

2) for every $x \in \mathcal{X}$ and every $u \in \mathcal{U}$ the set $C(x, u)$ is precompact, open and convex such that $x \in C(x, u)$

3) for every $x \in \mathcal{X}$ and every $u \in \mathcal{U}$ the set $\tilde{f}_{k}(x, u, \mathcal{X})$ is convex,

4) the state evolution (1a) is described by: if $\tilde{f}_{k}\left(x_{k}, u_{k}, w_{k}\right) \in C\left(x_{k}, u_{k}\right)$

$$
x_{k+1}=\tilde{f}_{k}\left(x_{k}, u_{k}, w_{k}\right)
$$

otherwise

$$
x_{k+1}=\partial C\left(x_{k}, u_{k}\right) \cap \mathcal{R}^{0}\left(x_{k}, \tilde{f}\left(x_{k}, u_{k}, w_{k}\right)\right),
$$

where $\mathcal{R}^{0}$ is defined by

$$
\mathcal{R}^{0}\left(x_{k}, x\right):=\left\{x_{k}+t\left(x-x_{k}\right): t \geq 0\right\} .
$$

Note that from the properties of convex sets, the intersection in (4b) contains exactly one element. Thus, $x_{k+1}$ is uniquely defined. There are other possibilities to define the projections than it is done in (4b) (e.g., projection on the closest point on the boundary). The projection approach that we have chosen is motivated by the fact that the discretizations of piecewise linear continuous systems would have such intersections as defined by (4b). The bounds $\left\{C\left(x_{k}, u_{k}\right)\right\}_{k=0}^{+\infty}$ of a CSSDS form a (possibly unbounded) stochastic process taking values in $\Sigma_{\mathcal{X}}$. Also, it is worth noting that when the 
system is not saturated functions $\tilde{f}_{k}$ and $f_{k}$ are equal. To help understand the meaning of (4)-(5) we have illustrated a possible trajectory of the stochastic processes $\left\{x_{k}\right\}_{k=0}^{+\infty}$ and $\left\{C\left(x_{k}, u_{k}\right)\right\}_{k=0}^{+\infty}$ in Figure 1.

We are interested in continuous state space, therefore it is reasonable to assume that for every time step $k$ the random variable $\tilde{f}_{k}\left(x_{k}, u_{k}, w_{k}\right)$ has a continuous PDF. This, however, does not hold for the variables $x_{k}$. Indeed, from (4) it follows that each variable $x_{k+1}$ has singularities at the boundary of $C\left(x_{k}, u_{k}\right)$. This means that the PDF of $x_{k+1}$ is continuous in the interior of the set $C\left(x_{k}, u_{k}\right)$ and has discontinuities at the boundary $\partial C\left(x_{k}, u_{k}\right)$. However, as we will see in Section III, the PDF of $x_{k+1}$ restricted to $\partial C\left(x_{k}, u_{k}\right)$ is a continuous function.

By (4)-(5) the conditional density of the variable $x_{k+1}$ given the previous state $x_{k}$ and input $u_{k}$ is given by:

$\mathbb{P}\left(x_{k+1}=x \mid x_{k}, u_{k}\right)=$

$\mathbb{P}\left(\tilde{f}_{k}\left(x_{k}, u_{k}, w_{k}\right)=x \mid x_{k}, u_{k}\right) \mathbf{1}_{C\left(x_{k}, u_{k}\right)}(x)$

$+\left[\int_{\mathcal{R}^{1}\left(x_{k}, x\right)} \mathbb{P}\left(\tilde{f}_{k}\left(x_{k}, u_{k}, w_{k}\right)=z \mid x_{k}, u_{k}\right) d H_{1}(z)\right] \mathbf{1}_{\partial C\left(x_{k}, u_{k}\right)}(x)$,

where $1_{A}(\cdot)$ denotes the indicator function of a set $A, H_{n}$ denotes the $n$-dimensional Hausdorff measure [24], and $\mathcal{R}^{1}$ is a set defined by

$$
\mathcal{R}^{1}\left(x_{k}, x\right):=\left\{x_{k}+t\left(x-x_{k}\right): t \geq 1\right\} .
$$

Hausdorff measure can be seen as a generalization of the Lebesgue measure to general metric spaces $(X, \rho)$. It is used to assign a "proper" measure to low dimensional objects embedded into higher dimensional space (e.g., 1dimensional Hausdorff measure of a curve embedded into $\mathbb{R}^{d}$ with metric $\rho$ measures the curve's length with respect to $\rho$ ). For any set $D \subset X$ the $n$-dimensional Hausdorff measure of $D$ is defined by:

$H_{n}(D):=\lim _{\delta \rightarrow 0} \inf \left\{\sum_{i=1}^{+\infty}\left(\operatorname{diam}\left(U_{i}\right)\right)^{n}: D \subset \bigcup_{i=1}^{+\infty} U_{i}, \operatorname{diam}\left(U_{i}\right)<\delta\right\}$.

Figure 2 plots $\mathcal{R}^{0}\left(x_{k}, \tilde{f}_{k}\left(x_{k}, u_{k}, w_{k}\right)\right)$ versus $\mathcal{R}^{1}\left(x_{k}, x\right)$.

A comparison of a PDF of an unsaturated variable $\tilde{f}_{k}\left(x_{k}, u_{k}, w_{k}\right)$ with the PDF of a saturated variable $x_{k+1}$ is presented in Figure 3.

Having the CSSDS defined in such a way, we are interested in estimating the actual state $x_{k}$ of the system from the available measurements $y_{k}$. The Markovian character of the CSSDS makes it possible, for estimation purposes, to employ recursive algorithms utilizing Bayes' theorem, e.g., the PF.

There are many variations of PFs [8], which employ various importance densities and resampling algorithms. For the sake of comparison, as a benchmark solution to the estimation problem suitable to saturated systems we chose the Constrained Bootstrap Particle Filter (CBPF), i.e., the Bootstrap Particle Filter (BPF) [8] modified by projecting

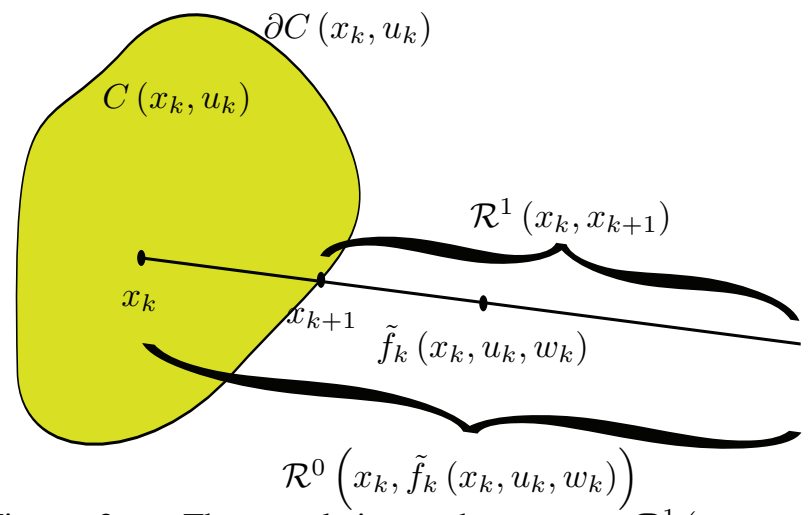

Fig. 2: The relation between $\mathcal{R}^{1}\left(x_{k}, x_{k+1}\right)$ and $\mathcal{R}^{0}\left(x_{k}, \tilde{f}_{k}\left(x_{k}, u_{k}, w_{k}\right)\right)$ when the saturated state of the system $x_{k+1} \in \partial C\left(x_{k}, u_{k}\right)$ is obtained by projecting the unsaturated state of the system $\tilde{f}_{k}\left(x_{k}, u_{k}, w_{k}\right)$ onto the saturation boundary $\partial C\left(x_{k}, u_{k}\right)$.

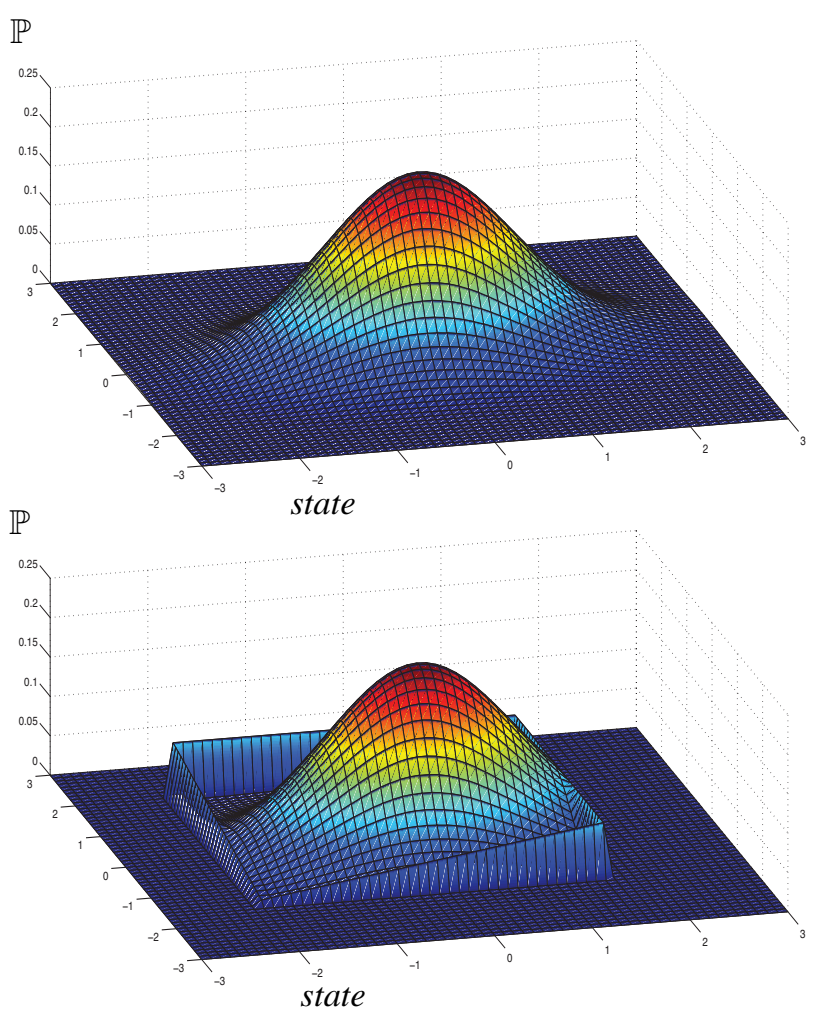

Fig. 3: The PDF of the unsaturated variable $\tilde{f}\left(x_{k}, u_{k}, w_{k}\right)$ (above) and the PDF of the saturated variable $x_{k+1}$ (below) given the previous state $x_{k}$ and input $u_{k}$. The PDF of the unsaturated variable is continuous (above) whereas the PDF of the saturated variable (below) has a continuous part (6a) and a singular mass (6b) concentrated on the saturation region $\partial C\left(x_{k}, u_{k}\right)=\partial([-2,1.5] \times[-1.5,2])$.

the particles violating the state constraints onto the boundary before the resampling takes place, which is an approach described in [12]. 


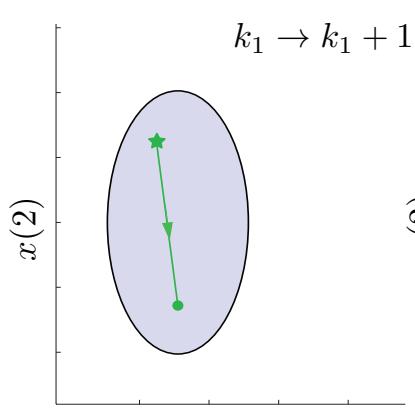

$x(1)$

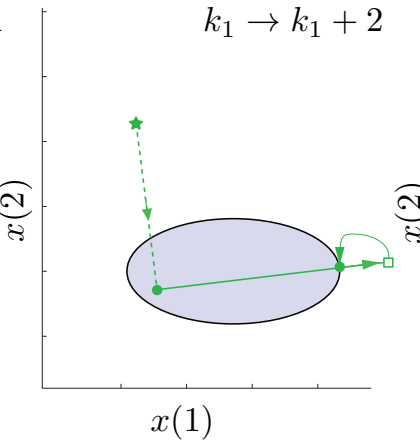

$x(1)$

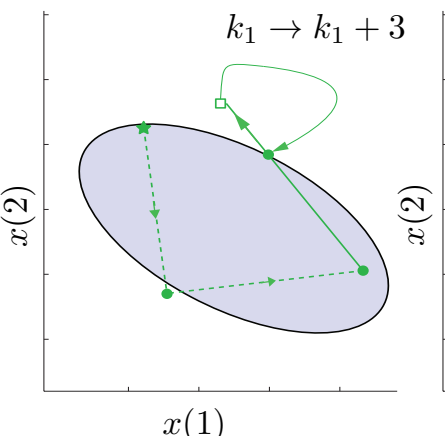

$x(1)$

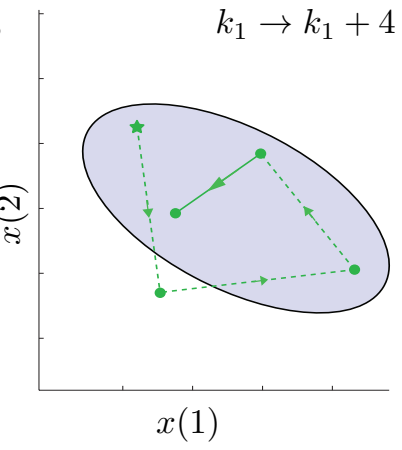

Fig. 1: Evolution of a two dimensional CSSDS $\left\{x_{k}\right\}_{k=0}^{+\infty}$, from $x_{k_{1}}$ (star) to $x_{k_{1}+4}$ (dots) and its constraint regions $\left\{C\left(x_{k}, u_{k}\right)\right\}_{k=0}^{+\infty}$ (shaded ellipses). When the unsaturated variable $\tilde{f}_{k}\left(x_{k}, u_{k}, w_{k}\right)$ (empty squares) exceeds the saturation boundary $\partial C\left(x_{k}, u_{k}\right)$ it is projected on the appropriate point (dots) at the saturation boundary.

\section{B. Particle Filtering}

The PF is a Monte Carlo-type algorithm that represents the estimated PDF of the state $x_{k}$ by $N$ random samples (particles) $\left\{x_{k}^{i}\right\}_{i=1}^{N}$ with their associated (normalized) weights $\left\{\omega_{k}^{i}\right\}_{i=1}^{N}$ that are generated using the Sequential Importance Sampling (SIS) method [25], [8].

These pairs approximate the true PDF by:

$$
\mathbb{P}\left(x_{k}=x \mid y_{k}\right) \approx \sum_{i=1}^{N} \omega_{k}^{i} \delta_{0}\left(x-x_{k}^{i}\right),
$$

where $\delta_{0}$ denotes the Dirac delta at zero.

The SIS is a recursive algorithm that uses the most recent observation $y_{k}$ to compute $\left\{\left(x_{k}^{i}, \omega_{k}^{i}\right)\right\}_{i=1}^{N}$ in two steps. First, for every $i=1, \ldots, N$, a sample $x_{k}^{i}$ is drawn from a (chosen) importance density $\mathbb{Q}\left(\cdot \mid x_{k-1}^{i}, u_{k-1}, y_{k}\right)$. Next, using the most recent observation $y_{k}$, the weights $\omega_{k}^{i}$ are updated according to the Bayes' rule

$\tilde{\omega}_{k}^{i}=\omega_{k-1}^{i} \frac{\mathbb{P}\left(h_{k}\left(x_{k}, v_{k}\right)=y_{k} \mid x_{k}^{i}\right) \mathbb{P}\left(x_{k}=x \mid x_{k-1}^{i}, u_{k-1}\right)}{\mathbb{Q}\left(x_{k}^{i} \mid x_{k-1}^{i}, u_{k-1}, y_{k}\right)}$

and normalized

$$
\omega_{k}^{i}=\frac{\tilde{\omega}_{k}^{i}}{\sum_{j=1}^{N} \tilde{\omega}_{k}^{j}} .
$$

On the practical side, a common problem of PFs is the particle degeneracy: after several iterations, all but few particles will have negligible weights. When that occurs most of the computational power is wasted on updating negligible weights and the accuracy of the algorithm strongly deteriorates since the true pdf is approximated only by a small set of significant particles. The degeneracy phenomenon can be circumvented by the appropriate resampling of the particles [2], [21], [26], [27].

The best possible importance density is the true posterior pdf itself [25]. However, in general, it is impossible to sample from it and hence, in practice one needs to rely on suboptimal importance densities. A popular approach, utilized by the BPF [9], [28], [29], is to use the transition density as an importance density:

$$
\mathbb{Q}\left(x \mid x_{k-1}^{i}, u_{k-1}, y_{k}\right):=\mathbb{P}\left(x_{k}=x \mid x_{k-1}^{i}, u_{k-1}\right) .
$$

Such a choice of the importance density is particularly appealing because, due to (12), the weight update (10) is simplified to:

$$
\tilde{\omega}_{k}^{i}=\omega_{k-1}^{i} \mathbb{P}\left(h_{k}\left(x_{k}, v_{k}\right)=y_{k} \mid x_{k}=x_{k}^{i}\right) .
$$

However, this comes with a price, namely that the most recent measurement $y_{k}$ is not used during the drawing of the particle.

In case of saturated processes the measurement $y_{k}$ can provide information of crucial importance, thus its loss during sampling is highly undesirable. The need of a special type of importance density that exploits a specific structure of the CSSDS and also accounts for the latest measurement $y_{k}$ led to the development of the SPF [17], [21]. The SPF was derived for a special class of CSSDSs, namely, for systems that allow only one-dimensional saturation [17]. In the next section we generalize it to multidimensional CSSDSs.

\section{CONVEX SATURATED PARTICLE FILTER}

In this section we propose a new SPF that is designed for CSSDSs. The CSPF is capable of quickly detecting whether or not saturation occurred by comparing the measurements with the state constraints. This information is used to forcibly move the particles to the region of higher probability, which leads to improved accuracy of the estimate. This procedure renders possible the reduction of the number of particles used by PF, thus reducing the computational load of the algorithm. The detection of the saturation is achieved by a detection function introduced in Definition 4.

Definition 4 (Detection function): A function $\alpha: \mathcal{Y} \times$ $\mathcal{X} \times \Sigma_{\mathcal{X}} \rightarrow \mathbb{R}$ is called a detection function for a CSSDS if for every time step $k$, every precompact open convex set $A \in$ 
$\Sigma_{\mathcal{X}}$, every $y \in \mathcal{Y}$ and $x_{1}, x_{2} \in \partial A$ the implication holds:

$$
\begin{aligned}
& \left\|y-h_{k}\left(x_{1}\right)\right\| \geq\left\|y-h_{k}\left(x_{2}\right)\right\| \Longrightarrow \\
& \alpha\left(y, x_{1}, h_{k}(A)\right) \leq \alpha\left(y, x_{2}, h_{k}(A)\right) .
\end{aligned}
$$

Intuitively, a function for which the condition (14) holds true serves as a 'pseudo'-metric between the points at the boundary $\partial A$ of the convex set $A$ and, through observation model $h_{k}$, the measurement $y \in \mathcal{Y}$. Note that in the definition of the detection function $\alpha$ in it not required that the inverse of the measurement function $h_{k}$ exists. Some indication on how this function should be tuned can be found in experimental results presented[17], [21], [30]. The properties of the detection functions are further explained while discussing the numerical example in Section IV.

Let us now consider the SSDS defined by (1)-(4). Furthermore, let $\left\{\left(x_{k}^{i}, \omega_{k}^{i}\right)\right\}_{i=1}^{N}$ be the approximation of the true PDF of the state of the process at time step $k$. For each $i \in\{1, \ldots, N\}$, given the previous particle $x_{k}^{i}$, the probability that the particle $x_{k+1}^{i}$ will saturate, i.e., $x_{k+1}^{i} \in$ $\partial C\left(x_{k}^{i}, u_{k}\right)$ follows from $(6 \mathrm{~b})$ :

$\mathbb{P}\left(x_{k+1}^{i} \in \partial C\left(x_{k}^{i}, u_{k}\right)\right)=$

$\int_{\partial C\left(x_{k}^{i}, u_{k}\right)} \int_{\mathcal{R}^{1}\left(x_{k}^{i}, x\right)} \mathbb{P}\left(\tilde{f}_{k}\left(x_{k}^{i}, u_{k}, w_{k}\right)=z \mid x_{k}^{i}, u_{k}\right) d H_{1}(z) d H_{n_{x}-1}(x)$

where $\mathcal{R}^{1}$ is defined by (7).

For the ease of notation the right-hand side of (15) is called the predicted probability of saturation and denoted as $q_{i}$, i.e.,

$$
q_{i}:=\int_{\partial C\left(x_{k}^{i}, u_{k}\right)} q_{i}(x) d H_{n_{x}-1}(x),
$$

where $q_{i}(\cdot)$ is defined by:

$q_{i}(x):=\int_{\mathcal{R}^{1}\left(x_{k}^{i}, x\right)} \mathbb{P}\left(\tilde{f}_{k}\left(x_{k}^{i}, u_{k}, w_{k}\right)=z \mid x_{k}^{i}, u_{k}\right) d H_{1}(z)$.

The saturation of a particle $x_{k+1}^{i}$ can be seen as projecting $x_{k+1}^{i}$ onto $\partial C\left(x_{k}^{i}, u_{k}\right)$ which is equivalent to the generic projection approach of [12]. Indeed, it makes no difference whether the 'bad' particles drawn from an unconstrained continuous distribution are projected on the saturation region, or each particle is set to saturation region with the predicted probability of saturation. The resulting sets of particles are equivalent in the statistical sense.

Let $\alpha$ be a given detection function satisfying Definition 4. Furthermore, assume that the measurement $y_{k+1}$ becomes available. Then, for each $i \in\{1, \ldots, N\}$ and each $x \in \partial C\left(x_{k}^{i}, u_{k}\right)$ we define the updated probability of saturation $q_{i}^{\alpha}$ :

$$
q_{i}^{\alpha}:=\int_{\partial C\left(x_{k}^{i}, u_{k}\right)} q_{i}^{\alpha}(x) d H_{n_{x}-1}(x),
$$

where

$$
q_{i}^{\alpha}(x) \propto q_{i}(x)+\alpha\left(y_{k+1}, x, C\left(x_{k}^{i}, u_{k}\right)\right) .
$$

Using (16)-(17), and the detection function $\alpha$, we define the importance density $\mathbb{Q}^{\alpha}$ of the new CSPF by:

$$
\begin{aligned}
& \mathbb{Q}^{\alpha}\left(x \mid x_{k}^{i}, u_{k}, y_{k+1}\right):=q_{i}^{\alpha}(x) \mathbf{1}_{\partial C\left(x_{k}^{i}, u_{k}\right)}(x) \\
& +\frac{1-q_{i}^{\alpha}}{1-q_{i}} \mathbb{P}\left(\tilde{f}_{k}\left(x_{k}, u_{k}, w_{k}\right)=x \mid x_{k}^{i}, u_{k}\right) \mathbf{1}_{C\left(x_{k}^{i}, u_{k}\right)}(x) .
\end{aligned}
$$

It can be easily seen that $\mathbb{Q}^{\alpha}$ defines a probability measure, i.e., $\mathbb{Q}^{\alpha}$ is positive, and it integrates to one. The importance density of the CBPF filter is a special case of $\mathbb{Q}^{\alpha}$ with $\alpha \equiv 0$.

Given the particle $x_{k}^{i}$ and the input $u_{k}$, a new particle $x_{k+1}^{i}$ is drawn from the importance density $\mathbb{Q}^{\alpha}$. The random sample form $\mathbb{Q}^{\alpha}$ is obtained in two steps. First, the algorithm determines whether the particle $x_{k+1}^{i}$ saturates, i.e., $x_{k+1}^{i} \in \partial C\left(x_{k}^{i}, u_{k}\right)$ (with the probability $q_{i}^{\alpha}$ ) or not (with probability $1-q_{i}^{\alpha}$ ). Next, if the saturation was detected the particle $x_{k+1}^{i}$ is drawn from:

$$
x_{k+1}^{i} \sim \frac{q_{i}^{\alpha}(\cdot)}{q_{i}^{\alpha}} \mathbf{1}_{\partial C\left(x_{k}^{i}, u_{k}\right)}(\cdot) .
$$

In case the saturation was not detected the particle $x_{k+1}^{i}$ is drawn from:

$$
x_{k+1}^{i} \sim \frac{1}{1-q_{i}} \mathbb{P}\left(\tilde{f}_{k}\left(x_{k}, u_{k}, w_{k}\right)=\bullet \mid x_{k}^{i}\right) \mathbf{1}_{C\left(x_{k}^{i}, u_{k}\right)}(\cdot) .
$$

The associated weights $\omega_{k+1}^{i}$ are derived from the general principle (10) applied to the importance density (18). If $x_{k+1}^{i}$ saturates then, by (16) and (17), the weight $\omega_{k+1}^{i}$ is given by:

$\omega_{k+1}^{i} \propto \omega_{k}^{i} \frac{q_{i}\left(x_{k+1}^{i}\right)}{q_{i}^{\alpha}\left(x_{k+1}^{i}\right)} \mathbb{P}\left(h_{k+1}\left(x_{k+1}, v_{k+1}\right)=y_{k+1} \mid x_{k+1}^{i}\right)$,

if $x_{k+1}^{i}$ does not saturate, the weight $\omega_{k+1}^{i}$ is updated by:

$$
\omega_{k+1}^{i} \propto \omega_{k}^{i} \frac{1-q_{i}}{1-q_{i}^{\alpha}} \mathbb{P}\left(h_{k+1}\left(x_{k+1}, v_{k+1}\right)=y_{k+1} \mid x_{k+1}^{i}\right) .
$$

The new CSPF is summarized in Algorithm 1.

The proposed CSPF combines the previous state $x_{k}^{i}$ with the most recent measurement $y_{k+1}$ to compute the updated probabilities of saturation $q_{i}^{\alpha}(x)$ and the total updated probability of saturation $q_{i}^{\alpha}$. The function

$$
\partial C\left(x_{k}^{i}, u_{k}\right) \ni x \mapsto \frac{q_{i}^{\alpha}(x)}{q_{i}^{\alpha}},
$$

is a continuous PDF on $\partial C\left(x_{k}^{i}, u_{k}\right)$.

Note that since each particle $x_{k}^{i}$ has dynamics of its own, for $i \neq j$ the constraint regions $C\left(x_{k}^{i}, u_{k}\right)$ and $C\left(x_{k}^{j}, u_{k}\right)$ do not need to overlap. As a consequence it is possible that the $i$-th saturation region $\partial C\left(x_{k}^{i}, u_{k}\right)$ nontrivially intersects with the admissible (unsaturated) part of the $j$-th constraint region $C\left(x_{k}^{j}, u_{k}\right)$. Thus, a region of the state space that is admissible to all the particles $\left\{x_{k}^{i}\right\}_{i}$ is given by $\bigcap_{i} C\left(x_{k}^{i}, u_{k}\right)$, and is also convex.

For large values of $q_{i}^{\alpha}$ the algorithm forces the particles to be close to the saturation region $\partial C\left(x_{k}^{i}, u_{k}\right)$ associated to the $i$-th particle, whereas for small values of $q_{i}^{\alpha}$ the particles are set further from the saturation region. Moreover, the 


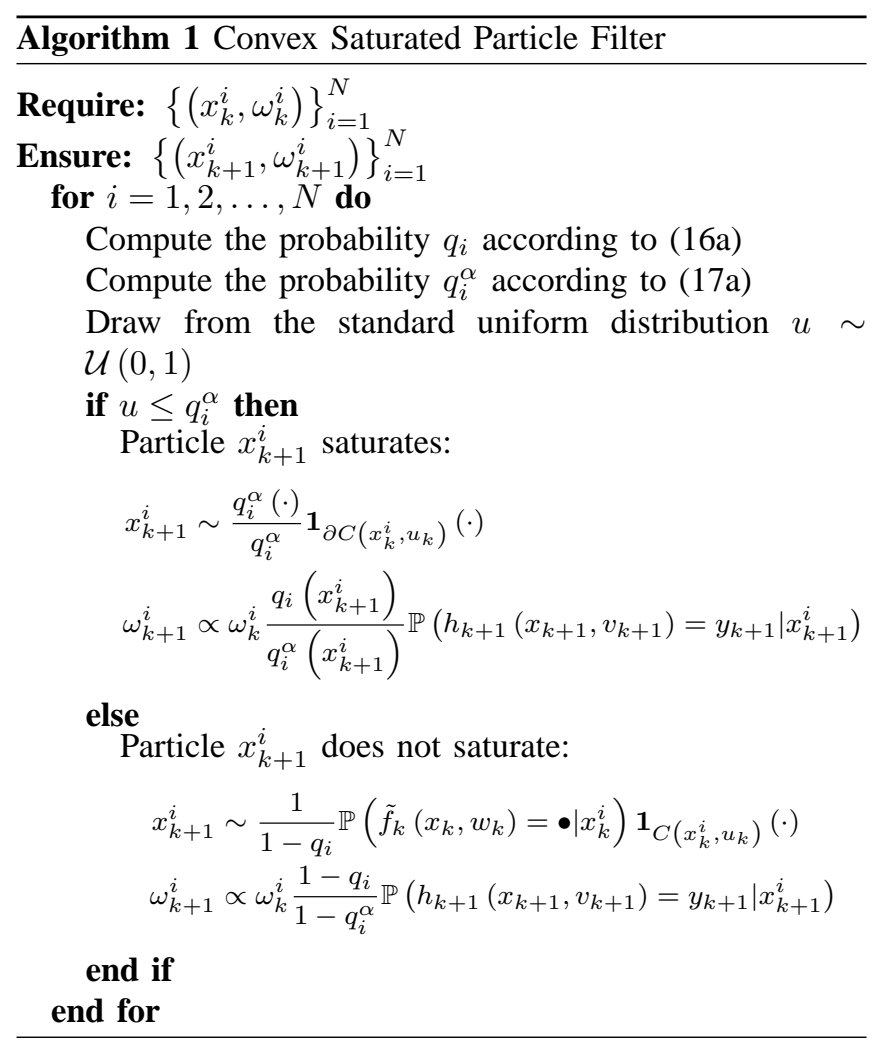

particles that hit the boundary of the constraint region, thanks to (23), are forcibly moved to that part of the saturation region that has the highest probability. Figure 4 schematically describes the differences between the Unconstrained BPF, the CBPF and the CSPF for a large value of $q_{i}^{\alpha}$.

Finally, we have to mention that the accuracy of the CSPF estimate depends on the user-specified detection function, which must be chosen appropriately to the CSSDS under consideration. This is a subject of ongoing research.

\section{NUMERICAL SIMULATIONS}

To illustrate the estimation abilities of the newly proposed CSPF we compare it with the CBPF applied to a simple CSSDS that models the motion of a two-dimensional object under random disturbance. We assume a static sensor placed at the origin that measures the distance and the bearing of the moving object. This model is a version of a classical nonlinear tracking problem discussed, e.g., in [28], [11], [31]. In this motivating example we discuss in detail how to overcome the difficulties of practical implementations of the CSPF that arise from the extra integrations steps (16)(17).

We consider the unconstrained system defined by:

$$
\begin{gathered}
{\left[\begin{array}{l}
x(1) \\
x(2)
\end{array}\right]_{k+1}=\left[\begin{array}{l}
x(1) \\
x(2)
\end{array}\right]_{k}+T_{s}\left[\begin{array}{l}
u(1) \\
u(2)
\end{array}\right]_{k}+w_{k},} \\
{\left[\begin{array}{l}
y(1) \\
y(2)
\end{array}\right]_{k}=\left[\begin{array}{c}
\sqrt{(x(1))^{2}+(x(2))^{2}} \\
\arctan \frac{x(2)}{x(1)}
\end{array}\right]_{k}+v_{k}}
\end{gathered}
$$

\section{I: UBPF}

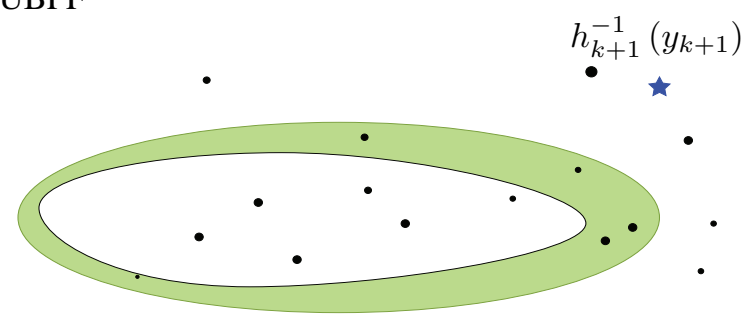

II: CBPF

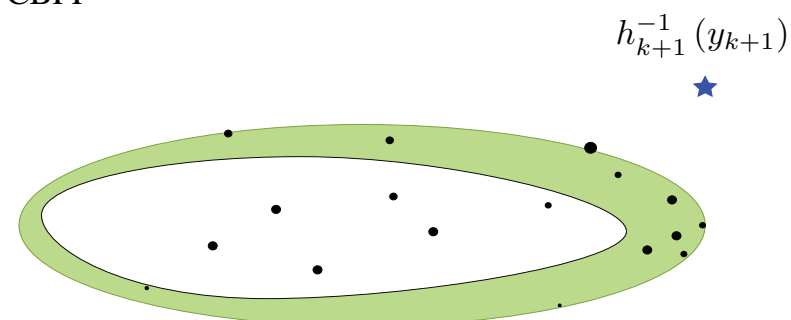

III: CSPF

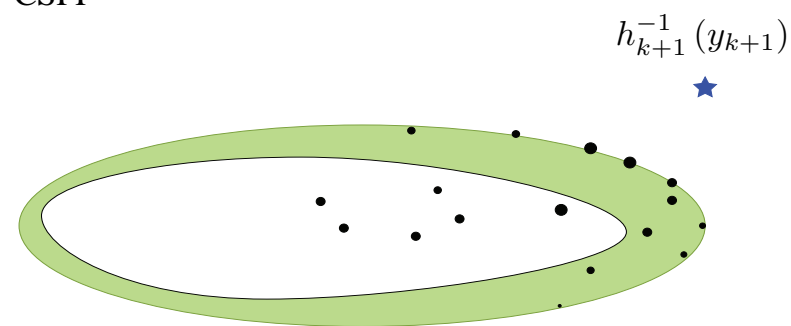

Fig. 4: Visualization of the distribution of particles obtained by the Unconstrained BPF (top), CBPF (middle) and the CSPF (bottom). Some of the particles obtained by the Unconstrained BPF violate the physical constraints $\bigcup_{i} C\left(x_{k}^{i}, u_{k}\right)$ of the system (shaded area and the region $\bigcap C\left(x_{k}^{i}, u_{k}\right)$ bounded by it), others are located far from the actual measurement (star). The larger the size of a particle the higher its weight. The CBPF projects the unphysical particles onto the relevant saturation regions (inside the shaded area), but does not move the remaining particles. The CSPF projects the 'bad' particles onto the saturation region and forces the remaining particles to concentrate closer to the saturation region. Moreover, weights of the repositioned particles are appropriately rescaled.

where $w_{k}$ and $v_{k}$ are two-dimensional zero-mean Gaussian variables with the covariance matrices $\Sigma_{x}=\left[\begin{array}{ll}5 & 0 \\ 0 & 5\end{array}\right]$ and $\Sigma_{y}=\left[\begin{array}{cc}0.1 & 0 \\ 0 & 0.0012\end{array}\right]$ respectively. The controlled input $u$ corresponds to the velocity of the object and $T_{s}=1$ is the sampling period. The constraint imposed on such a system is defined by

$$
\begin{aligned}
& C\left(x_{k}, u_{k}\right):= \\
& \left\{x:\left(x_{k}+T_{s} u_{k}-x\right)^{T}\left(x_{k}+T_{s} u_{k}-x\right) \leq r^{2}\left\|u_{k}\right\|^{2}\right\},
\end{aligned}
$$

where $r$ is the user-specified parameter that, in our simulation, is set to $r=2$. Thus, the constraint region $C\left(x_{k}, u_{k}\right)$ is a ball centered in $x_{k}+u_{k}$ with radius $r\left\|u_{k}\right\|$. 
The detection function $\alpha$ for the CSPF measures the difference between the distances between the inverse measurement $h_{k+1}^{-1}\left(y_{k+1}\right)$ (where $h_{k}$ denotes the standard polar transformation) and the particle $x_{k}^{i}+u_{k}$ and the vector at the boundary of the constraint region $C\left(x_{k}^{i}, u_{k}\right)$ :

$$
\begin{aligned}
& \alpha\left(y_{k+1}, x, C\left(x_{k}^{i}, u_{k}\right)\right):=\theta\left\|h_{k+1}^{-1}\left(y_{k+1}\right)-x_{k}^{i}-T_{s} u_{k}\right\| \times \\
& \left(\left\|h_{k+1}^{-1}\left(y_{k+1}\right)-x_{k}^{i}-T_{s} u_{k}\right\|-\left\|h_{k+1}^{-1}\left(y_{k+1}\right)-x\right\|\right),
\end{aligned}
$$

for $x \in \partial C\left(x_{k}^{i}, u_{k}\right)$, and user-specified parameter $\theta$. The strength of the influence of the detection function (26) depends on the value of the parameter $\theta$. We can see that the function defined by (26) satisfies the condition (14). In an extreme situation, when $\theta=0$, the function $\alpha$ has zero influence on the algorithm and the CSPF degenerates into the CBPF.

With the help of a function defined by (26) it is now possible to update the probability of saturation of all the particles $x_{k+1}^{i}$ by comparing the distances between the inverse measurement $h_{k+1}^{-1}\left(y_{k+1}\right)$ and the points at the boundary of the constraint region $C\left(x_{k}^{i}, u_{k}\right)$.

To show how such an update can be achieved we need to consider two situations

1) If the inverse observation $h_{k+1}^{-1}\left(y_{k+1}\right)$ belongs to the interior $C\left(x_{k}^{i}, u_{k}\right)$, then for all the points at the boundary $x \in \partial C\left(x_{k}^{i}, u_{k}\right)$ the updated probability of saturation $q_{i}^{\alpha}$

- increases, if $\left\|x-h_{k+1}^{-1}\left(y_{k+1}\right)\right\|$ $\left\|h_{k+1}^{-1}\left(y_{k+1}\right)-x_{k}^{i}-u_{k}\right\|$

- decreases, if $\left\|x-h_{k+1}^{-1}\left(y_{k+1}\right)\right\|>$ $\left\|h_{k+1}^{-1}\left(y_{k+1}\right)-x_{k}^{i}-u_{k}\right\|$.

2) For the inverse observation $h_{k+1}^{-1}\left(y_{k+1}\right)$ that belongs to $\mathcal{X} \backslash C\left(x_{k}^{i}, u_{k}\right)$ the updated probability of saturation $q_{i}^{\alpha}$

- increases, if $\left\|x-h_{k+1}^{-1}\left(y_{k+1}\right)\right\| \leq r\left\|u_{k}\right\|+$ $\operatorname{dist}\left(h_{k+1}^{-1}\left(y_{k+1}\right), C\left(x_{k}^{i}, u_{k}\right)\right)$,

- decreases, if $\left\|x-h_{k+1}^{-1}\left(y_{k+1}\right)\right\|>r\left\|u_{k}\right\|+$ $\operatorname{dist}\left(h_{k+1}^{-1}\left(y_{k+1}\right), C\left(x_{k}^{i}, u_{k}\right)\right)$.

To help understand the properties of the probabilities of saturation, the second of the discussed cases is visualized in Figure 5.

In order to successfully implement the CSPF for the model (24)-(25) we need to tackle the following technical problems:

1) numerically evaluate the integrals (16),

2) numerically evaluate the integral (17),

3) draw random samples from the distribution $\frac{q_{i}^{\alpha}(\cdot)}{q_{i}^{\alpha}}$.

The first problem is solved in Proposition 1. The points $2 \& 3$ are closely connected hence are dealt with together.

Proposition 1: The predicted probability of saturation $q_{i}(\cdot)$ for the system defined by (24)-(25) is uniformly distributed on the circle $\partial C\left(x_{k}^{i}, u_{k}\right)$ and it integrates to

$$
q_{i}=1-\frac{1}{\Sigma_{11}} \int_{0}^{r\left\|u_{k}\right\|} \exp \left(-\frac{z^{2}}{2 \Sigma_{11}}\right) d z
$$

where $\Sigma_{11}$ denotes the first diagonal entry of the matrix $\Sigma_{x}$.

Proof: The uniform distribution of $q_{i}(\cdot)$ follows directly from the fact that circles $\partial C\left(x_{k}^{i}, u_{k}\right)$ coincide with the $\sigma$ contours of the Gaussian variable $w_{k}$.

Formula (27) can be easily obtained by the polar parametrization of the plane $\mathbb{R}^{2}$ and by observing that

$$
\mathbb{P}\left(x_{k+1} \in \partial C\left(x_{k}, u_{k}\right)\right)=1-\mathbb{P}\left(x_{k+1} \in C\left(x_{k}, u_{k}\right)\right) .
$$

The probability in (28) is easily computed by:

$$
\begin{aligned}
& \mathbb{P}\left(x_{k+1} \in C\left(x_{k}, u_{k}\right)\right) \\
& =\frac{1}{2 \pi\left|\Sigma_{x}\right|^{1 / 2}} \int_{C\left(x_{k}, u_{k}\right)} \exp \left(-\frac{1}{2} x^{T} \Sigma_{x}^{-1} x\right) d x \\
& =\frac{1}{2 \pi \Sigma_{11}} \int_{0}^{2 \pi} \int_{0}^{r\left\|u_{k}\right\|} \exp \left(-\frac{z^{2}}{2 \Sigma_{11}}\right) d z s \phi \\
& =\frac{1}{\Sigma_{11}} \int_{0}^{r\left\|u_{k}\right\|} \exp \left(-\frac{z^{2}}{2 \Sigma_{11}}\right) d z .
\end{aligned}
$$

Combining (28) with (29c) gives the desired (27).

To compute integral (16) it is convenient to introduce the following parametrization of the boundary of the constraint region:

$$
\begin{aligned}
& \partial C\left(x_{k}, u_{k}\right)= \\
& \left\{x_{k}+u_{k}+r\left\|u_{k}\right\|(\cos (\phi), \sin (\phi)): \phi \in[0,2 \pi)\right\} .
\end{aligned}
$$

Then the updated probability of saturation (17b) can be seen as a function defined on the interval $[0,2 \pi)$ :

$$
[0,2 \pi) \ni \phi \mapsto q_{i}^{\alpha}(x(\phi)),
$$

hence the integral (17a) can be efficiently evaluated, e.g., with the MATLAB function quad.m.

The parametrization (30) is further used to obtain samples from $\frac{q_{i}^{\alpha}(\cdot)}{q_{i}^{\alpha}}$ through the use of the inverse of a Cumulative Density Function (CDF). The procedure of obtaining such a 'pseudo'-random sample is described in Algorithm 2.

Note, that $\operatorname{CDF}(\phi)$ is continuous and monotone function that can be easily evaluated for any given $\phi$. Thus, a solution to $\operatorname{CDF}\left(\phi_{0}\right)=u$ can be obtained by applying any of the standard root-finding algorithms [33].

Using the aforementioned numerical techniques, we have simulated the system (24) with the constraint (25) for $T=20$ time steps. We consider three different simulation scenarios to investigate various properties of the proposed CSPF:

1) Scenario I: robustness with respect to the initial condition of the filter,

2) Scenario II: robustness with respect to the detection function $\alpha$,

3) Scenario III: robustness with respect to the number of particles used by the filter.

All the algorithms were executed in Matlab 7.9 on a Mac OS X with an Intel Core 2 Duo $2.66 \mathrm{GHz}$ CPU with 4 GB RAM. 

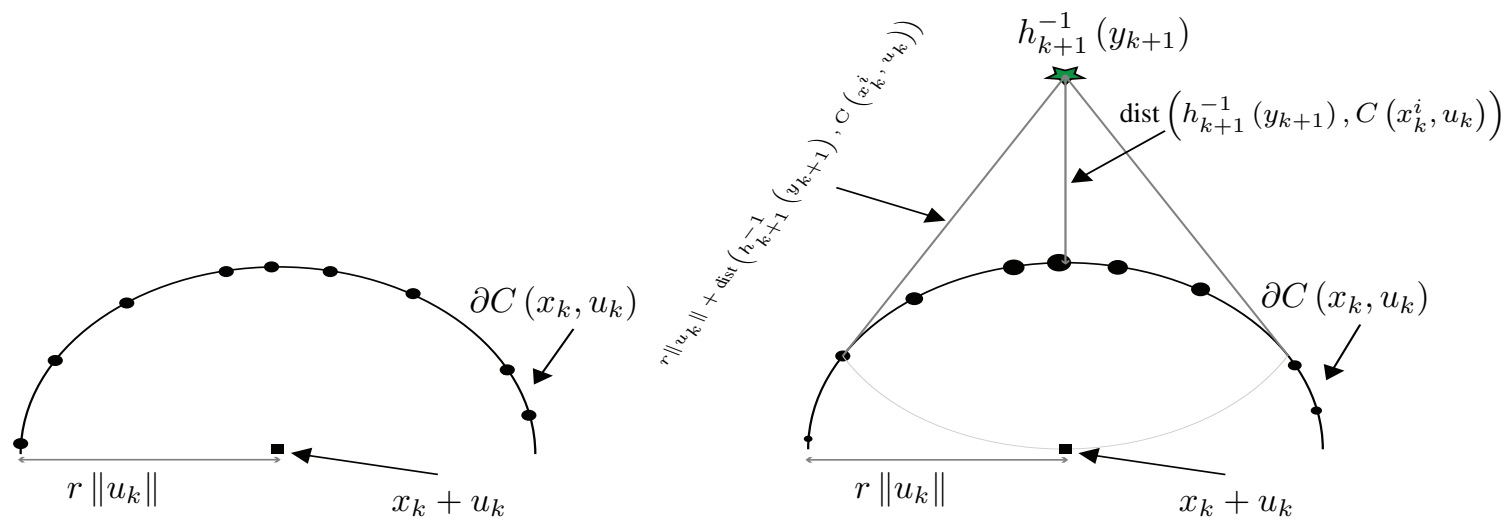

Fig. 5: The visualization of the updated probability of saturation obtained with the use of detection function (26). Before the measurement $y_{k+1}$ is collected (left) the probability of saturation is uniformly distributed on the boundary $\partial C\left(x_{k}, u_{k}\right)$. After the measurement $y_{k+1}$ becomes available (right) the probability of saturation is rescaled to account for the newest information.

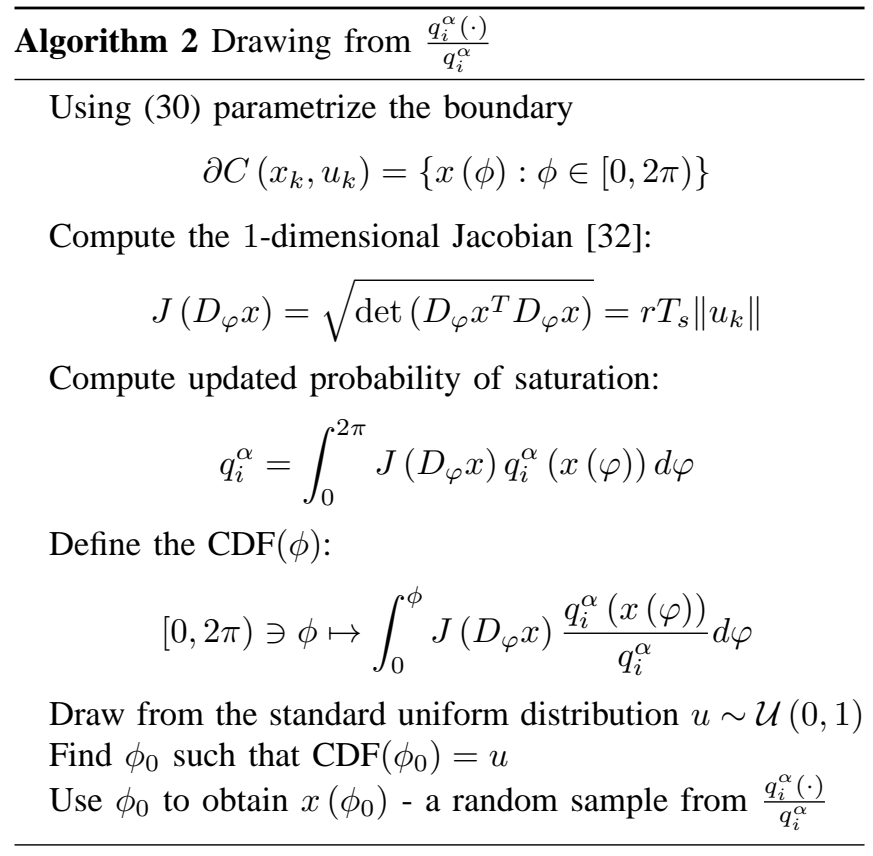

the CSPF ten times, each utilizing the same observation sequence. The average mean square error (AMSE) of both filters obtained for all three simulation scenarios are reported in Table I. Table II reports the average times required for the CBPF and the CSPF to produce a single-step estimate of the state.

Figure 6 reports the averages of ten independent runs of the CBPF and the CSPF each using 1000 particles. The estimates were obtained by the CBPF and the CSPF starting from initial positions: $\left[\begin{array}{ll}5 & 15\end{array}\right]^{T},\left[\begin{array}{ll}10 & 10\end{array}\right]^{T}$ and $\left[\begin{array}{ll}15 & 5\end{array}\right]^{T}$.

TABLE I: AMSE of the CBPF and the CSPF for Scenario I

\begin{tabular}{|c|c|c|c|}
\hline & \multicolumn{3}{|c|}{ Initial condition } \\
\hline & $x(0)=\left[\begin{array}{ll}15 & 5\end{array}\right]^{T}$ & $x(0)=\left[\begin{array}{ll}10 & 10\end{array}\right]^{T}$ & $x(0)=\left[\begin{array}{ll}5 & 15\end{array}\right]^{T}$ \\
\hline $\mathrm{CBPF}$ & 22.33 & 20.05 & 22.06 \\
$\mathrm{CSPF}$ & 5.91 & 3.71 & 5.88 \\
\hline
\end{tabular}

\section{A. Scenario I}

In the simulations discussed in this section the parameter in the detection function (26) is set to $\theta=\frac{0.005}{r\left\|u_{k}\right\|}$ for appropriate scaling. The simulation started from the initial condition $x_{0}=\left[\begin{array}{ll}10 & 10\end{array}\right]^{T}$ and the input signal $u_{k}=\left[\begin{array}{ll}3 & 3\end{array}\right]^{T}$ is constant over the whole simulation.

We tested the CBPF and the CSPF in three settings that differ with the initial condition used by both filters. Namely, we simulated the case with no initial offset $x_{0}^{\text {filters }}=$ $\left[\begin{array}{ll}10 & 10\end{array}\right]^{T}$, and two scenarios with initial offsets: $x_{0}^{\text {filters }}=$ $\left[\begin{array}{ll}5 & 15\end{array}\right]^{T}$ and $x_{0}^{\text {filters }}=\left[\begin{array}{ll}15 & 5\end{array}\right]^{T}$. In each of the aforementioned settings the CBPF and the CSPF use the initial covariance $P_{0}=\left[\begin{array}{ll}1 & 0 \\ 0 & 1\end{array}\right]$. Both filters use 1000 particles.

To account for the probabilistic nature of the compared methods in every simulation setting we run the CBPF and

TABLE II: Average computational times (in seconds) of the CBPF and the CSPF for Scenario I

\begin{tabular}{|c|c|c|c|}
\hline & \multicolumn{3}{|c|}{ Initial condition } \\
\hline & $x(0)=\left[\begin{array}{ll}15 & 5\end{array}\right]^{T}$ & $x(0)=\left[\begin{array}{ll}10 & 10\end{array}\right]^{T}$ & $x(0)=\left[\begin{array}{ll}5 & 15\end{array}\right]^{T}$ \\
\hline $\mathrm{CBPF}$ & 0.2122 & 0.2030 & 0.2318 \\
$\mathrm{CSPF}$ & 11.6219 & 12.1172 & 12.7369 \\
\hline
\end{tabular}

\section{B. Scenario II}

In the simulations discussed in this section we have tested three different parameters in the detection function (26): $\theta=$ $\frac{0.005}{r\left\|u_{k}\right\|}, \theta=\frac{0.05}{r\left\|u_{k}\right\|}$, and $\theta=\frac{0.5}{r\left\|u_{k}\right\|}$. The simulation started from the initial condition $x_{0}=\left[\begin{array}{ll}5 & -5\end{array}\right]^{T}$ and the input signal $u_{k}=\left[\begin{array}{ll}3 & 3\end{array}\right]^{T}$ is constant over the whole simulation. 

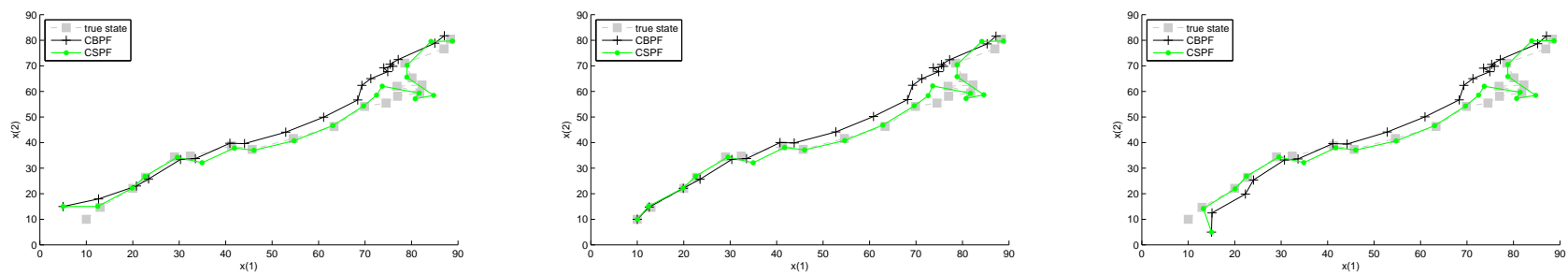

Fig. 6: Scenario I. Trajectory of the true target (filled squares) and the estimates computed by the CBPF (solid line with pluses) and by the CSPF (solid-dotted line) in two dimensional plane. The estimates are obtained as the average of ten independent filters each utilizing 1000 particles. The estimates were obtained by the CBPF and the CSPF starting from initial positions: $\left[\begin{array}{ll}5 & 15\end{array}\right]^{T}$ (left), $\left[\begin{array}{ll}10 & 10\end{array}\right]^{T}$ (middle) and $\left[\begin{array}{ll}15 & 5\end{array}\right]^{T}$ (right).

There is no offset in the initial conditions for the filters. In each of the aforementioned settings the CBPF and the CSPF use the initial covariance $P_{0}=\left[\begin{array}{ll}1 & 0 \\ 0 & 1\end{array}\right]$. Both filters use 10 particles.

Similarly as in the previous section in every simulation from this section we run the CBPF and the CSPF ten times, each utilizing the same observation sequence. The AMSE of both filters obtained for all three simulation scenarios are reported in Table III. Table IV reports the average times required for the CBPF and the CSPF to produce a singlestep estimate of the state. Figure 7 reports the averages of ten independent runs of the CBPF and the CSPF each using 10 particles.

TABLE III: AMSE of the CBPF and the CSPF for Scenario II

\begin{tabular}{|l|c|c|c|}
\hline & \multicolumn{3}{|c|}{ Value of the parameter $\theta$} \\
\hline$x(0)=\left[\begin{array}{ll}5 & -5\end{array}\right]^{T}$ & $\theta=\frac{0.005}{r\left\|u_{k}\right\|}$ & $\theta=\frac{0.05}{r\left\|u_{k}\right\|}$ & $\theta=\frac{0.5}{r\left\|u_{k}\right\|}$ \\
\hline $\mathrm{CBPF}$ & 28.3694 & 27.2028 & 28.3694 \\
$\mathrm{CSPF}$ & 6.0381 & 2.8517 & 1.5484 \\
\hline
\end{tabular}

TABLE IV: Average computational times (in seconds) of the CBPF and the CSPF for Scenario II

\begin{tabular}{|c|c|c|c|}
\hline & \multicolumn{3}{|c|}{ Value of the parameter $\theta$} \\
\hline & $\theta=\frac{0.005}{r\left\|u_{k}\right\|}$ & $\theta=\frac{0.05}{r\left\|u_{k}\right\|}$ & $\theta=\frac{0.5}{r\left\|u_{k}\right\|}$ \\
\hline $\mathrm{CBPF}$ & 0.004 & 0.004 & 0.004 \\
$\mathrm{CSPF}$ & 0.1306 & 0.1648 & 0.2154 \\
\hline
\end{tabular}

\section{Scenario III}

In the simulations discussed in this section the parameter in the detection function (26) is constant and is set to $\theta=$ $\frac{0.005}{r\left\|u_{k}\right\|}$. The simulation started from the initial condition $x_{0}=$ $\left[\begin{array}{ll}-15 & -5\end{array}\right]^{T}$ and the input signal $u_{k}=\left[\begin{array}{ll}3 & 3\end{array}\right]^{T}$ is again set constant over the whole simulation.
We examined the CBPF and the CSPF using different number of particles: 10, 100, and 1000. There is no offset in the initial condition of the filters and the initial covariance is again set to $P_{0}=\left[\begin{array}{ll}1 & 0 \\ 0 & 1\end{array}\right]$

The CBPF and the CSPF are both run ten times, each utilizing the same observation sequence. The AMSE corresponding to this scenario are shown in Table $\mathrm{V}$ and the average times required for the CBPF and the CSPF to produce a single-step estimate of the state are given in Table VI.

Figure 8 presents the averages of ten independent runs of the CBPF and the CSPF using: 10, 100 and 1000 particles.

TABLE V: AMSE of the CBPF and the CSPF for Scenario III

\begin{tabular}{|l|c|c|c|}
\hline & \multicolumn{3}{|c|}{ Number of particles } \\
\hline$x(0)=\left[\begin{array}{ll}-15 & -5\end{array}\right]^{T}$ & $N=10^{1}$ & $N=10^{2}$ & $N=10^{3}$ \\
\hline $\mathrm{CBPF}$ & 1438 & 796 & 267 \\
$\mathrm{CSPF}$ & 6.8302 & 1.8049 & 1.6583 \\
\hline
\end{tabular}

TABLE VI: Average computational times (in seconds) of the CBPF and the CSPF for Scenario III

\begin{tabular}{|c|c|c|c|}
\hline & \multicolumn{3}{|c|}{ Number of particles } \\
\hline & $N=10^{1}$ & $N=10^{2}$ & $N=10^{3}$ \\
\hline $\mathrm{CBPF}$ & 0.0034 & 0.0265 & 0.2191 \\
$\mathrm{CSPF}$ & 0.1406 & 1.3972 & 14.1950 \\
\hline
\end{tabular}

\section{CONCLUSIONS}

In this paper we extended the previously proposed estimation method, the SPF, which makes an effective use of the measurements during the importance sampling, to multidimensional SSDS. Such extension requires an extra condition to be imposed on the system, namely the constraints of the system need to be convex sets in $\mathbb{R}^{n}$. With the convexity 

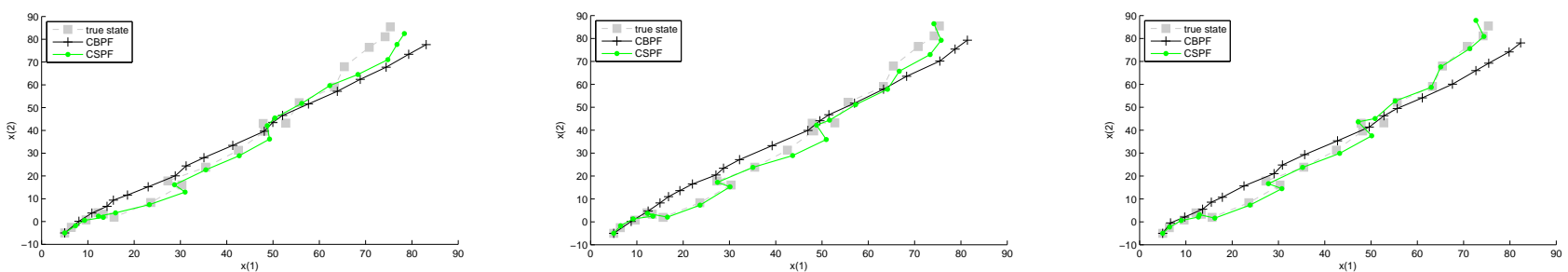

Fig. 7: Scenario II. Trajectory of the true target (filled squares) and the estimates computed by the CBPF (solid line with pluses) and by the CSPF (solid-dotted line) in two dimensional plane. The estimates are obtained as the average of ten independent filters each utilizing 10 particles. The estimates were obtained by the CBPF and the CSPF starting from the initial position $\left[\begin{array}{ll}5 & -5\end{array}\right]^{T}$. The CSPF utilize different detection functions (26) with parameters: $\theta=\frac{0.005}{r\left\|u_{k}\right\|}$ (left), $\theta=\frac{0.05}{r\left\|u_{k}\right\|}$ (middle) and $\theta=\frac{0.5}{r\left\|u_{k}\right\|}$ (right).
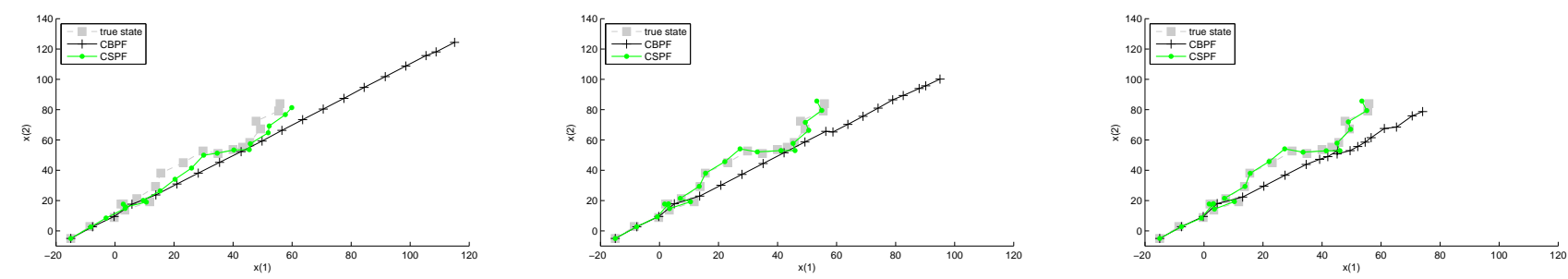

Fig. 8: Scenario III. Trajectory of the true target (filled squares) and the estimates computed by the CBPF (solid line with pluses) and by the CSPF (solid-dotted line) in two dimensional plane. The estimates are obtained as the average of ten independent filters each starting from the initial position $\left[\begin{array}{ll}-15 & -5\end{array}\right]^{T}$. The estimates were obtained by the CBPF and the CSPF utilizing: 10 particles (left), 100 particles (middle) and 1000 particles (right).

assumption satisfied, the multidimensional detection function can be properly defined. This function is then used to derive the multidimensional CSPF that utilizes the measurements to detect the saturation of the system while sampling new particles.

We demonstrated the efficiency of our new method by applying the CSPF to the two-dimensional motion model with linear unconstrained dynamics, bounded disturbances, and nonlinear noisy measurements. The simulations suggest that for the concerned system the CSPF is as robust with respect to the choice of initial condition as the CBPF is. This can be seen in Figure 6 and Table I. Simulations depicted in Figure 7 and summarized in Table III suggest that appropriate choice of the detection function can further improve the performance of the CSPF. Furthermore, in the performed simulations the CSPF achieves accurate performance using much fewer particles than the CBPF use, which can be observed Figure 8 and Table V.

In our simulations the CSPF outperforms the benchmark CBPF in terms of speed of convergence to the true signal from the initial offset as well as the accuracy of tracking the signal with saturated dynamics. This can be observed in Figures 6, 7, and 8 that report the outcomes of the CSPF and the CBPF.

The improved performance of the CSPF comes with the price of increased computational time required by the new filter, which on average is approximately 58 times slower than the CBPF. This is due to computationally expensive procedures specific for the CSPF that involve evaluation of the integral (17a) and drawing samples according to Algorithm 2. However, as it is reported in simulation Scenario III and in Table V the CSPF using only 10 particles achieves accuracy comparable or even better than the accuracy achieved by the CBPF with 1000 particles. This means that for the CSPF, the family of particles might be kept small which greatly reduces the computational effort of the algorithm. Thus, given that the computational complexity of both compared filters grows linearly with the number of particles used, which can be seen in Table II, we can argue that the CSPF outperforms the benchmark CBPF in both accuracy and computational time.

As could been observed in Figure 8, the performance of the filter depends on the appropriate selection of the detection function. The optimal choice of the detection function is connected to the construction of a proper resampling algorithm that yields desirable asymptotic properties of the CSPF. These topics where not discussed in current paper as they are still a matter of ongoing research.

\section{ACKNOWLEDGEMENT}

This research is funded by the dredging company IHC Systems B. V., P. O. Box 41, 3360 AA Sliedrecht, the Netherlands. 


\section{REFERENCES}

[1] R. Kalman, "A New Approach to Linear Filtering and Prediction Problems," Transctions of the ASME - Journal of Basic Engineering, vol. 82, pp. 35-45, 1960.

[2] B. Ristic, M. S. Arulampalam, and N. Gordon, Beyond the Kalman Filter: Particle Filters for Tracking Application. Artech House, 2004.

[3] S. C. Patwardhan, J. Prakash, S. Narasimhan, R. B. Gopaluni, and S. L. Shah, "Nonlinear Bayesian State Estimation: Review and Recent Trends," Control Engineering Practice, vol. 20(10), pp. 933 - 953, 2012.

[4] S. Julier and J. Uhlmann, "A General Method for Approximating Nonlinear Transformations of Probability Distributions," Robotics Research Group, Department of Engineering Science, University of Oxford, Tech. Rep., 1996.

[5] — "Unscented Filtering and Nonlinear Estimation," Proceedings of the IEEE, vol. 92, pp. 401-422, 2004.

[6] K. Ito and K. Xiong, "Gaussian Filters for Nonlinear Filtering Problems," IEEE Transactions on Automatic Control, vol. 45, pp. 910-927, 2000.

[7] I. Arasaratnam, S. Haykin, and R. Elliott, "Discrete-Time Nonlinear Filtering Algorithms Using Gauss-Hermite Quadrature," Proceedings of the IEEE, vol. 95, pp. 953-977, 2007.

[8] S. Arulampalam, S. Maskell, N. Gordon, and T. Clapp, "A Tutorial on Particle Filters for Online Nonlinear/Non- Gaussian Bayesian Tracking," IEEE Transactions on Signal Processing, vol. 50, pp. 174188, 2002.

[9] D. Cristian and A. Doucet, "A Survey of Convergence Results on Particle Filtering Methods for Practitioners," IEEE Transactions on Signal Processing, vol. 50, pp. 736-746, 2002.

[10] B. P. Carlin, N. G. Polson, and D. S. Stoffer, "A Monte Carlo Apraoch to Nonnormal and Nonlinear State-Space Models," Journal of the American Statistical Association, vol. 87, pp. 493-500, 1992.

[11] W. R. Gilks and C. Berzuini, "Following a Moving Target - Monte Carlo Inference for Dynamic Bayesian Models," Journal of the Royal Statistical Society, vol. 63, pp. 127-146, 2001.

[12] X. Shao, B. Huang, and J. M. Lee, "Constrained Bayesian State Estimation - A Comparative Study and a New Particle Filter Based Approach," Journal of Process and Control, vol. 20, pp. 143-157, 2010.

[13] L. Lang, W. Chen, B. R. Bakshi, P. K. Goel, and S. Ungarala, "Bayesian Estimation via Sequential Monte Carlo Sampling - Constrained Dynamic Systems," Automatica, vol. 43, pp. 1615-1622, 2007.

[14] J. Prakash, S. C. Patwardhan, and S. L. Shah, "Constrained Nonlinear State Estimation Using Ensemble Kalman Filters," Industrial and Engineering Chemistry Research, vol. 49, pp. 2242-2253, 2010.

[15] O. Straka, J. Dunik, and M. Simandl, "Truncated Unscented Particle Filter," in Proceedings of the American Control Conference, San Francisco, California, 2011, pp. 1825-1830.

[16] I. Kyriakides, D. Morrel, and A. Papandreou-Suppappola, "A Particle Filtering Approach to Constrained Motion Estimation in Tracking Multiple Targets," in The 39th Asilomar Conference on Signals, Systems and Computers, vol. 28, Pacific Grove, California, 2005, pp. 94-98.

[17] P. Stano, Zs. Lendek, and R. Babuška, "Saturated Particle Filters," in Proceedings of the American Control Conference, San Francisco, California, 2011, pp. 1819-1824.

[18] P. M. Stano, Zs. Lendek, J. Braaksma, R. Babuška, and C. de Keizer, "Particle Filters for Estimating Average Grain Diameter of Material Excavated by Hopper Dredger," in Proceedings of IEEE Conference on Control Applications, Yokohama, Japan, 2010, pp. 292 - 297.

[19] P. Vachhani, S. Narasimhan, and R. Rengaswamy, "Robust and Reliable Estimation via Unscented Recursive Nonlinear Dynamic Data Reconciliation," Journal of Process Control, vol. 16, pp. 1075-1086, 2006.

[20] W. Stadje, "A New Approach to the Lindley Recursion," Statistics and Probability Letters, vol. 31, pp. 169-175, 1997.

[21] P. M. Stano, Zs. Lendek, and R. Babuška, "Saturated Particle Filter: Almost Sure Convergence and Improved Resampling," Automatica, vol. 49 (1), pp. 1477-159, 2013.

[22] G. Dantzig, Linear Programming and Extensions. Princeton University Press, 1998

[23] J. M. Lee, Introduction to Topological Manifolds, ser. Graduate Texts in Mathematics. Springer, 2010.
[24] G. B. Folland, Real analysis: modern techniques and their applications, ser. Pure and applied mathematics. Wiley, 1984.

[25] A. Doucet, S. Godsill, and C. Andrieu, "On Sequential Monte Carlo Sampling Methods for Bayesian Filtering," Statistics and Computing, vol. 10, pp. 197-208, 2000.

[26] X. Fu and Y. Jia, "An Improvement on Resampling Algorithms of Particle Filters," IEEE Transactions on Signal Processing, vol. 58, pp. 5414-5420, 2010.

[27] D. S. Lee and N. K. K. Chia, "A Particle Algorithm for Sequential Bayesian Parameter Estimation and Model Selection," IEEE Transactions on Signal Processing, vol. 50, pp. 326-336, 2002.

[28] N. J. Gordon, D. J. Salmond, and A. F. M. Smith, "Novel Approach to Nonlinear/Non-Gaussian Bayesian State Estimation," IEEProceedings- $F$, vol. 140, pp. 107-113, 1993.

[29] G. Kitagawa, "Monte Carlo Filter and Smoother for Non-Gaussian Nonllinear State Space Models," Journal of Computational and Graphical Statistics, vol. 5, pp. 1-25, 1996.

[30] P. M. Stano, "Nonlinear state and parameter estimation for hopper dredgers," Ph.D. dissertation, Delft University of Technology,, 2013.

[31] M. S. Arulampalam, B. Ristic, N. Gordon, and T. Mansell, "BearingsOnly Tracking of Manoeuvring Targets Using Particle Filters," EURASIP Journal on Applied Signal Processing, vol. 15, pp. 23512365, 2004.

[32] S. G. Krantz and H. R. Parks, Geometric Integration Theory, ser Cornerstones Series. Birkhäuser Boston, 2008.

[33] W. H. Press, B. P. Flannery, S. A. Teukolsky, and W. T. Vetterling, Numerical Recipes in Fortran 77: The Art of Scientific Computing. New York, NY, USA: Cambridge University Press, 2003. 